\title{
Developing a predictive in vitro dissolution model based on gastrointestinal fluid characterisation in rats
}

Christfort, Juliane Fjelrad; Strindberg, Sophie ; Plum, Jakob; Hall-Andersen, Jonatan ; Janfelt, Christian; Nielsen, Line Hagner; Müllertz, Anette

\author{
Published in: \\ European Journal of Pharmaceutics and Biopharmaceutics
}

Link to article, DOI:

10.1016/j.ejpb.2019.07.007

Publication date:

2019

Document Version

Peer reviewed version

Link back to DTU Orbit

Citation (APA):

Christfort, J. F., Strindberg, S., Plum, J., Hall-Andersen, J., Janfelt, C., Nielsen, L. H., \& Müllertz, A. (2019). Developing a predictive in vitro dissolution model based on gastrointestinal fluid characterisation in rats. European Journal of Pharmaceutics and Biopharmaceutics, 142, 307-314. https://doi.org/10.1016/j.ejpb.2019.07.007

\section{General rights}

Copyright and moral rights for the publications made accessible in the public portal are retained by the authors and/or other copyright owners and it is a condition of accessing publications that users recognise and abide by the legal requirements associated with these rights.

- Users may download and print one copy of any publication from the public portal for the purpose of private study or research.

- You may not further distribute the material or use it for any profit-making activity or commercial gain

- You may freely distribute the URL identifying the publication in the public portal 


\section{Developing a predictive in vitro dissolution model based on gastrointestinal}

\section{2 fluid characterisation in rats}

3 Juliane Fjelrad Christfort ${ }^{\text {a,b }}$, Sophie Strindberg a , Jakob Plum ${ }^{\text {a,c }}$, Jonatan Hall-Andersen ${ }^{\text {a }}$, Christian

4 Janfelt ${ }^{\mathrm{a}}$, Line Hagner Nielsen ${ }^{\mathrm{b}}$, Anette Müllertz ${ }^{\mathrm{a}, \mathrm{d}, 1}$

5 a Department of Pharmacy, Faculty of Health and Medical Sciences, University of Copenhagen, DK-2100 Copenhagen, Denmark

6 b Department of Health Technology, Technical University of Denmark, DK-2800 Lyngby, Denmark

$7 \quad$ c Present address: LEO Pharma A/S, Industriparken 55, DK-2750 Ballerup, Denmark

$8 \quad{ }^{d}$ Bioneer:FARMA, Faculty of Health and Medical Sciences, University of Copenhagen, DK-2100 Copenhagen, Denmark

Keywords

11 Biorelevant media; gastrointestinal fluids; rats; in vitro in vivo correlation.

Abbreviations

ASSF, amorphous sodium salt of furosemide; GI, Gastrointestinal; HEPES, 4-(2-hydroxyethyl)-1piperazineethanesulfonic acid; IVIVC, in vitro in vivo correlation; MALDI, matrix-assisted laser desorption ionisation; MSI, mass spectrometry imaging; RGM, Rat gastric medium; RIM, Rat intestinal medium.

\section{Abstract}

The influence of physiological factors on the solubility of drug compounds has been thoroughly investigated in humans. However, as these factors vary between species and since many in vivo studies are carried out in rats or mice, it has been difficult to establish sufficient in vitro in vivo relations. The aim of this study was to develop a physiologically relevant in vitro dissolution model simulating the gastrointestinal (GI) fluids of fasted rats and compare it to previously published in vitro and in vivo data. To develop the in vitro model, the $\mathrm{pH}$ was measured in situ in six segments of the GI tract of anesthetised rats, then the fluids from the stomach, the proximal and the distal small intestine were collected and characterized with regard to osmolality, and bile acid and phospholipid

\footnotetext{
${ }^{1}$ Correspondence to: Anette Müllertz

Department of Pharmacy,

University of Copenhagen,

Universitetsparken 2

DK-2100 Copenhagen, Denmark

Telephone: 004535336440

E-mail address: anette.mullertz@sund.ku.dk
} 
concentration. The $\mathrm{pH}$ and osmolality were found to increase throughout the GI tract. The bile acids and phospholipids were present in high concentrations in the proximal small intestine, and the bile acid concentration doubled in the distal part, where the phospholipid concentration decreased. Matrixassisted laser desorption ionisation mass spectrometry imaging was applied on a cross section of the small intestine, to study which bile acids and phospholipid classes were present in the small intestine of rats. Both cholic acid, taurocholic acid and glycocholic acid were detected, and phosphatidylcholine (34:2) was found to be mainly present in the intestinal wall or mucus, whereas lysophosphatidylcholine (16:0) was also detected in the lumen. Based on these observations, biorelevant media were developed to simulate fluids in the stomach and the proximal part of the small intestine in fasted rats. The media were implemented in a two-step in vitro dissolution model, which was found to better predict the in vivo performance of furosemide, when compared to previously published in vitro and in vivo data.

\section{Introduction}

During the past years, there has been an intensified focus on how to improve the solubility of poorly water-soluble compounds to optimise their oral bioavailability. Many models are available to evaluate dissolution and solubility of poorly water-soluble compounds in vitro [1, 2]. However, currently available in vitro models have several times shown inability to predict oral preclinical in vivo pharmacokinetics of compounds with poor aqueous solubility [3-5]. For example, it was found in a recent study, that the in vitro dissolution of amorphous sodium salt of furosemide (ASSF) had a higher solubility and 20-fold faster dissolution rate compared to the crystalline acid form of furosemide. However, this did not translate into differences in the pharmacokinetic profile after oral dosing to rats [5]. 
As a step towards improvement of the IVIVC, great effort has been put into investigation of the conditions in the gastrointestinal (GI) tract of humans, to be able to perform the in vitro studies under conditions as close as possible to the in vivo situation [6-8]. Volume, $\mathrm{pH}$, osmolality, surface tension and surfactant concentration are some of the parameters known to affect the dissolution and solubility of a compound in the GI tract. For both fasted and fed conditions, several fluid compositions have been suggested as biorelevant media to simulate conditions in the human GI tract, and hence, improve IVIVC [9-11]. Multiple biorelevant media are also commercially available, based on the composition of GI fluids in humans and dogs [12]. However, since the composition of the GI fluids vary greatly between species, it is challenging to obtain good IVIVC for in vivo studies performed in commonly used laboratory animals, e.g. rats and mice. Thus, an approach to improve IVIVC is to obtain knowledge about the fundamental aspects of the GI tract of common preclinical species (e.g. rats), which can be translated into novel in vitro models.

Warnken et al. [13] investigated the capability of an in vitro dissolution/absorption method using simulated media based on human data to predict in vivo performance in mice. The authors found that especially the change in $\mathrm{pH}$ to a value relevant for the preclinical species improved the IVIVC [13]. Besides the $\mathrm{pH}$, also the concentration of bile acids and phospholipids has been found to vary between fasted GI fluids from rats and humans (Table 1). Values as $\mathrm{pH}$ and levels of bile acids and phospholipids not only vary from humans to rats, but also between studies of GI fluids from different breeds of fasted rats (Table 1). The current study will focus exclusively on values from fasted Sprague-Dawley rats, since fasted rats are commonly used for pharmacokinetic studies.

Table 1: Overview of studies evaluating $\mathrm{pH}$ and levels of bile acids, phospholipids and osmolality in the GI tract of fasted rats. Human values as recently reviewed by Bergström et al. [14] are included for comparison.

\begin{tabular}{llll}
\hline Parameter & & Rat (fasted) & Human (fasted) $^{\mathbf{d}}$ \\
\hline $\mathbf{p H}$ & Stomach & $\begin{array}{l}1.4-1.9^{\mathrm{a}}, 3.9^{\mathrm{b}}, 4.3 \text { (forestomach) }^{\mathrm{c}}, 4.0 \\
\text { (glandular stomach) }^{\mathrm{c}} \text {, germ free rats: } 3.8\end{array}$ & $1.7-3.3$ \\
& & $\begin{array}{l}\text { (forestomach) } \\
\text { ( }, 5.7 \text { (glandular stomach) }^{\mathrm{c}} .\end{array}$ & \\
& Proximal small intestine & $6.7-7.0^{\mathrm{a}}, 5.9-6.1^{\mathrm{b}}, 7.1^{\mathrm{c}}$ & $5.6-7.8$ \\
& Distal small intestine & $6.9-7.4^{\mathrm{a}}, 5.9^{\mathrm{b}}, 8.0^{\mathrm{c}}$ & - \\
\hline
\end{tabular}




\begin{tabular}{llll}
\hline $\begin{array}{l}\text { Bile acids } \\
\mathbf{( m M )}\end{array}$ & Stomach & $<5^{\mathrm{a}}$ & $0-0.8$ \\
& Proximal small intestine & $51^{\mathrm{a}}$ & $1.4-5.9$ \\
& Distal small intestine & $102^{\mathrm{a}}$ & - \\
\hline $\begin{array}{l}\text { Phospholipids } \\
\mathbf{( m M )}\end{array}$ & Stomach & hardly detected $^{\mathrm{a}}$ & 0.029 \\
& Proximal small intestine & $1.5-5.5^{\mathrm{a}}$ & $0.1-0.6$ \\
\hline Osmolality & Distal small intestine & hardly detected $^{\mathrm{a}}$ & - \\
$\mathbf{( m O s m / k g )}$ & Promach & - & $119-221$ \\
& Distal small intestine & - & $137-300$ \\
\hline
\end{tabular}

References: a: [15], b: [16], c: [17] and d: [14].

Furthermore, not only the amount, but also the type of bile acids and phospholipids are varying between species [18]. The most abundant bile acids in humans have recently been determined to be glyco-conjugates [19]. In contrast, Yang et al. [20] quantitatively investigated GI fluid from rats and found taurocholic acid to be the most abundant bile acid in the bile duct, whereas the unconjugated cholic acid was mainly present in the small intestine. However, more information is needed about the types of bile acids and phospholipids present in the GI tract of rats. One way to investigate this, is to use matrix-assisted laser desorption ionisation (MALDI) mass spectrometry imaging (MSI). MALDI MSI is a commonly used soft ionisation technique combining information about the molecular structure of an analyte with its location in the sample. It has previously been used to visualise drug distribution in tissue [21-23].

The aim of the current study was to investigate the total bile acid and phospholipid concentration, the osmolality and the $\mathrm{pH}$ in the stomach and small intestine of fasted rats, with the overall aim to produce gastric and intestinal biorelevant media simulating the composition of fasted rat GI fluid. In addition, the media were implemented in a setup currently used to study in vitro solubility, dissolution and drug release, to create a two-step model, simulating both gastric and intestinal conditions. Furthermore, an aim was to apply MALDI MSI to obtain detailed insight into which bile acid and phospholipid classes are most abundant in the proximal part of the small intestine in rats. 


\section{Materials and methods}

\subsection{Materials}

Crystalline furosemide acid, 2,5-dihydroxybenzoic acid (DHB) and taurocholic acid sodium salt hydrate were purchased from Sigma-Aldrich (St. Louis, MO, USA). Methanol, sodium chloride and chloroform were obtained from VWR (Radnor, PA, USA), where 4-(2-hydroxyethyl)-1piperazineethanesulfonic acid (HEPES) was acquired from Roth (Karlsruhe, Germany). Phosphatidylcholine (purity: $99.1 \%$ ) and lysophosphatidylcholine (purity: 80 \%), both from soybean oil, were kindly donated from Lipoid (Steinhausen, Schwitzerland). Purified water was provided from an Ultra Clear water system produced by Siemens (Munich, Germany).

\subsection{In situ $\mathrm{pH}$ measurements and sampling in anesthetised rats}

The rat study was carried out at the Department of Pharmacy, University of Copenhagen, under the license number 2016-15-0201-00892, and in compliance with Danish laws regulating experiments on animals and EU Directive 2010/63/EU.

Six male Sprague-Dawley rats (295 $\pm 9 \mathrm{~g}$ on the day of the experiment) were purchased from Janvier Labs (Le Genest-Saint-Isle, France) and were acclimatised for a minimum of 7 days on standard feed with free access to water under controlled environmental parameters (temperature: $22.1{ }^{\circ} \mathrm{C}$, relative humidity: $57 \%$ ), and with a reversed day/night rhythm (red light during the day and normal light during the night). Before starting the experiment, the animals were fasted for approximately $13 \mathrm{~h}$.

The rats were anesthetised subcutaneously with a mixture of Hypnorm (fentanyl $0.315 \mathrm{mg} / \mathrm{mL}$; fluanisone, $10 \mathrm{mg} / \mathrm{mL}$ )/Dormicum (midazolam, $5 \mathrm{mg} / \mathrm{mL}$ ), with a dose of $236 \mu \mathrm{g} / \mathrm{kg}$ fentanyl, 7.5 $\mathrm{mg} / \mathrm{kg}$ fluanisone and $3.75 \mathrm{mg} / \mathrm{kg}$ midazolam and placed on a $37^{\circ} \mathrm{C}$ heating pad to keep the body temperature constant. The abdominal wall was incised at linea alba and the stomach and the small intestine were located. The $\mathrm{pH}$ was measured in four segments of the GI tract; forestomach, glandular 


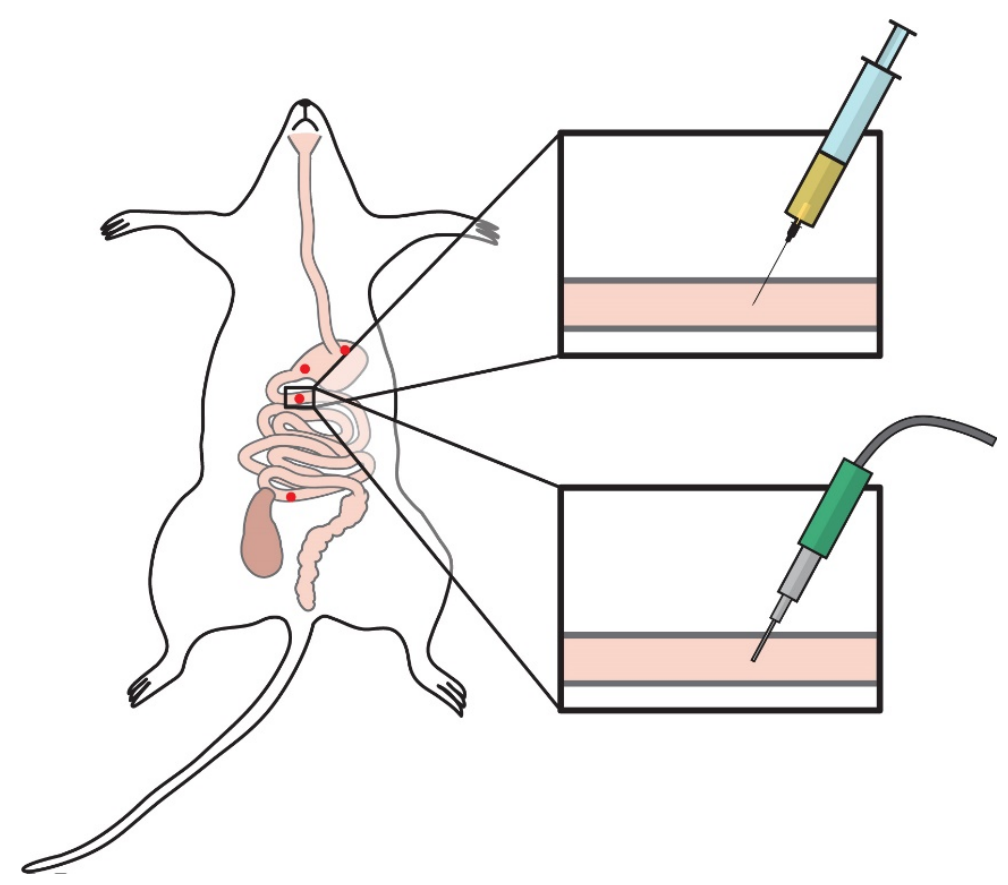

Figure 1: Schematic of the sampling and $\mathrm{pH}$ measurements in the GI tract of a rat. The investigated locations are illustrated by the red dots and correspond to; forestomach, glandular stomach, proximal small intestine ( 5 to $20 \mathrm{~cm}$ distal to the stomach) and distal small intestine (5 to $20 \mathrm{~cm}$ proximal to the caecum).

The $\mathrm{pH}$ was measured through a small incision in the different segments, using a micro $\mathrm{pH}$ electrode (Metrohm, Herisau, Schwitzerland) connected to a portable pH meter, PHM201 (Radiometer samples were collected with a syringe from the stomach, the proximal small intestine and the distal small intestine and transferred to polypropylene microtubes. All measurements and sampling were performed within 30 min after anesthesia for each rat. 
The osmolality of each sample was measured on the day of sampling on an Osmomat 030-D automatic cryoscopic osmometer (Gonotec, Berlin, Germany), and the samples were stored at $-20{ }^{\circ} \mathrm{C}$ until further analysis. The total concentrations of bile acids and phospholipids were determined with a fluorometric and colourimetric enzymatic assay kit, respectively (Sigma-Aldrich, MO, USA). The total concentration of bile acids was determined as the combined concentration of the twelve most common conjugated and unconjugated bile acids, whereas the total concentration of phospholipids was measured as the combined concentration of choline containing phospholipids in the samples. The fluids sampled from the GI tract of rats were centrifuged, and the supernatant was diluted with purified water to be within the range of the calibration curve for the kits. All standards and samples were measured on a multimode microplate reader according to the wavelengths in the protocol from the manufacturer (Thermo Fisher Scientific, MA, USA).

\subsection{MALDI MSI on sections from the small intestine}

The study was carried out under the license number and in compliance with the directives described in section 2.2. One male Sprague-Dawley rat (305 $\mathrm{g}$ on the day of the experiment) was used for studies of the distribution of bile acids and phospholipids in the small intestine. The rat was acclimatised and anaesthetised as described in section 2.2, but with a normal light cycle. The rat was fasted one hour prior to the experiment. The abdominal wall was incised at linea alba and the small intestine was located. The small intestine was cut out, immediately transferred to ice and stored at $80{ }^{\circ} \mathrm{C}$ until further analysis. The small intestine was cut in four segments $(0,10,20$ and $30 \mathrm{~cm}$ distal to the stomach) and pieces of the intestine were mounted on a cryo-microtome specimen plate using water as the only adhesive. The intestine was vertically mounted such that cross sections could be cut and mounted on glass slides. The intestine was cut in $25 \mu \mathrm{m}$ thin sections using a Leica CM3050S 
cryo-microtome (Leica Microsystems, Wetzlar, Germany), and the sections were thaw-mounted on glass slides, which were subsequently stored at $-80{ }^{\circ} \mathrm{C}$ until time of analysis.

At time of analysis, the samples were taken directly from $-80^{\circ} \mathrm{C}$ to a vacuum desiccator where they reached room temperature, thus avoiding condensation of water during thawing of the samples. The sample was coated by MALDI matrix by spraying a $30 \mathrm{mg} / \mathrm{mL}$ solution of 2,5-dihydroxybenzoic acid (dissolved in methanol and water, 70:30 v/v) using a setup described in details elsewhere [24].

The sample was analysed on a QExactive orbitrap mass spectrometer (Thermo Scientific, Bremen, Germany) equipped with the AP-SMALDI10 ion source (TransMIT GmbH, Giessen, Germany). Imaging was performed in the positive ion mode (scan range $m / z 230$ - 920) with a pixel size of 10$25 \mu \mathrm{m}$, and a DHB matrix peak was used as lock-mass for internal mass calibration, ensuring a mass accuracy of \pm 1 ppm or better. The raw file was converted to imzML using an imzML converter [25], and images were generated in MSiReader 1.01 [26] using a bin width of 6 ppm. To compensate for differences in ionisation efficiency across the sample, the intensities were normalised to the total ion current (TIC) of each individual mass spectrum. For each image, the colour scale was adjusted in order to enable details in the low-intensity regions of the images to appear clearly.

\subsection{Preparation of biorelevant media}

For preparation of the biorelevant media (Table 3), the required amount of a stock solution of lysophosphatidylcholine in chloroform (300 mM) was pipetted and a lipid film was formed by complete evaporation of the solvent under a steady stream of nitrogen. Sodium taurocholate and sodium chloride were added to achieve the desired bile acid concentrations and osmolalities and $\mathrm{HCl} / \mathrm{HEPES}$ was added according to Table 3 . The media were stirred overnight at $37^{\circ} \mathrm{C}$ and the next day the $\mathrm{pH}$ was adjusted to the desired value followed by addition of solvent to reach volume. The 
osmolality was measured using an Osmomat 030-D automatic cryoscopic osmometer (Gonotec, Berlin, Germany).

\subsection{Preparation of amorphous sodium salt of furosemide}

An ASSF was prepared as previously described by Nielsen et al. [5]. An amount of $0.4 \%$ w/v furosemide and $5 \mathrm{M} \mathrm{NaOH}$ (molar ratio of 1:1 with furosemide) was added to a solution of purified water and $96 \%$ ethanol $(10: 1 \mathrm{v} / \mathrm{v})$. The solution was spray dried and the obtained powder was stored in a glass vial protected from light.

\subsection{Dissolution of crystalline free acid and amorphous sodium salt of furosemide}

The dissolution of crystalline furosemide acid and ASSF in rat gastric medium (RGM) and and rat intestinal medium (RIM) was measured on a $\mu$ DISS Profiler ${ }^{\mathrm{TM}}$. The dissolution was measured as intrinsic dissolution as previously described by Nielsen et al. [5] in order to compare the dissolution curves. Briefly, experiments were performed at $37{ }^{\circ} \mathrm{C}$ with a stirring rate of $100 \mathrm{RPM}$, and the path length of the UV probes were $1 \mathrm{~mm}$. For the dissolution experiments, small discs of the two furosemide formulations were prepared using a mini-IDR tablet compression system (Heath Scientific, Milton Keynes, U.K.). Each disc comprised of 5 - $8 \mathrm{mg}$ of either of the furosemide forms (compressed at 80 bar for $40 \mathrm{~s}$ ). The disc loaded with furosemide was inserted into a cylindrical magnetic stirring bar, transferred to a sample vial and covered with $10 \mathrm{~mL}$ biorelevant medium (Table 3). UV measurements were carried out every $10 \mathrm{~s}$ with a total run time of 30 min under gastric conditions and $3 \mathrm{~h}$ and 33 min under intestinal conditions. A wavelength range of $310-350 \mathrm{~nm}$ was used to determine the concentration of dissolved furosemide. Furthermore, the dissolution of crystalline furosemide acid was evaluated in the developed two-step model (Section 3.4). All experiments were performed in $3-5$ replicates. 


\subsection{Data analysis}

Boxplots from the in situ rat studies are shown as minimum value, median and maximum value, and $25 \%$ and $75 \%$ percentiles were included where $n>3$. Thus, the osmolality, bile acid and phospholipid levels are reported as medians. For other tables and graphs, the results are shown as mean \pm standard deviation (SD).

Visualisation and statistical analysis were carried out in GraphPad Prism version 6.0 (GraphPad software Inc., La Jolla, CA, USA). Statistical differences $(\mathrm{p}<0.05)$ were determined using one-way ANOVA with a Tukey’s multiple comparison test.

\section{Results and discussion}

In the present study the rats were fasted during the inactive part of their cycle. Rats are nocturnal animals, i.e. they are active and eating during the night. This was reversed by acclimatising them in normal light during the night and red light during the day. For fasted state pharmacokinetic studies, rats are often fasted during their active period (overnight under normal light conditions), which can lead to stress symptoms and aggressive behaviour during handling. Furthermore, it has previously been observed by ex vivo examination, that the stomach of the rats was filled with bedding material after fasting during the active part of their cycle (data not published). All six rats included in the present study appeared healthy and non-stressed after fasting in the inactive part of their cycle, and no content was observed in the stomach at the beginning of the experiment.

In the previously published study by Nielsen et al. [5], which was used to evaluate the in vitro in vivo relation of the developed in vitro model, the rats were fasted overnight without a reversed day/night cycle. Although this is different from the fasting method used in the present study, it was decided to apply the reversed day/night cycle for ethical reasons and for the wellbeing of the animals. 


\subsection{In situ pH measurements}

227 The $\mathrm{pH}$ values were measured in the forestomach, glandular stomach, proximal and distal small 228 intestine of the rats (Table 2).

Table 2: $\mathrm{pH}$ values measured in situ in the GI tract of rats (mean $\pm \mathrm{SD}, \mathrm{n}=4-6$ ).

\begin{tabular}{ccccc}
\hline GI section & Forestomach & $\begin{array}{c}\text { Glandular } \\
\text { stomach }\end{array}$ & $\begin{array}{c}\text { Proximal } \\
\text { small intestine }\end{array}$ & $\begin{array}{c}\text { Distal } \\
\text { small intestine }\end{array}$ \\
\hline $\mathrm{pH}$ & $1.9 \pm 0.3$ & $2.9 \pm 0.7$ & $7.5 \pm 0.3$ & $7.8 \pm 0.3$ \\
\hline
\end{tabular}

The $\mathrm{pH}$ in the forestomach was measured to be $1.9 \pm 0.3$, which was significantly lower $(\mathrm{p}<0.05)$ than $\mathrm{pH}$ in the glandular stomach $(2.9 \pm 0.7)$. For all the individual rats, a similar significant rank order between $\mathrm{pH}$ in the forestomach and glandular stomach was seen, implying that the large SD occurs from inter-individual variations. The higher $\mathrm{pH}$ in the glandular stomach was not expected since the parietal cells responsible for acid secretion are located in this part of the stomach [27], thus, a more acidic environment would be expected in this area. In literature, only one study has investigated the $\mathrm{pH}$ separately in both the forestomach and the glandular stomach of fasted rats (both conventional and germ free), where the gastric $\mathrm{pH}$ values in germ free rats (Table 1) followed trends similar to the current study.

In the small intestine, the $\mathrm{pH}$ was measured to be $7.5 \pm 0.3$ in the proximal part, whereas it increased to be $7.8 \pm 0.3$ in the distal part (Table 2). In literature, similar $\mathrm{pH}$ values have been found after in situ measurements in the proximal and distal part of the small intestine in fasted rats (Table 1).

\subsection{Bile acids, phospholipids and osmolality}

246 The levels of bile acids, phospholipids and osmolality were determined in the stomach, proximal and distal small intestine (Fig. 2). A bile acid concentration of $1.3 \mathrm{mM}$ was measured in the stomach (Fig. 
the small intestine [27], but it could be due to reflux from the small intestine to the stomach. This phenomenon has previously been observed in humans [14]. In the small intestine, the bile acid concentration was found to be $24.1 \mathrm{mM}$ in the proximal part and increasing to $54.6 \mathrm{mM}$ in the distal part (Fig. 2A). Phospholipids were hardly detected in the stomach (0.8 mM) (Fig. 2B), resulting in a bile acid/phospholipid ratio of 1.6. The concentration of phospholipids was determined to be $3.7 \mathrm{mM}$ and $0.4 \mathrm{mM}$ in proximal and distal part, respectively. Hence, this results in bile acid/phospholipid ratios of 6.5 in the proximal part and 137 in the distal part of the small intestine. The osmolality in the stomach, proximal and distal small intestine was determined to be 230, 312 and $328 \mathrm{mOsm} / \mathrm{kg}$, respectively (Fig. 2C).
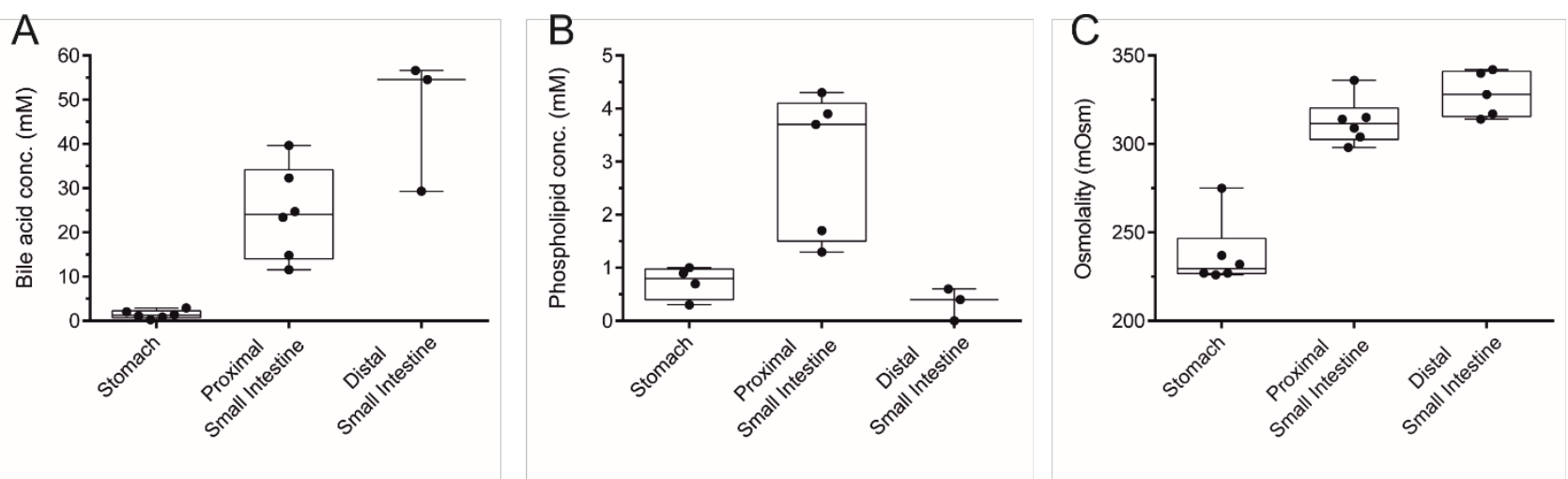

Figure 2: Boxplots showing the concentration of A: bile acids, B: phospholipids and C: the osmolality measured in the stomach and proximal and distal small intestine of rats. Results are shown as individual measurements, minimum value, median and maximum value, and $25 \%$ and $75 \%$ percentiles were included were $n>3(n=3-6)$.

A similar study found comparable phospholipid concentrations in fasted rats, but two times higher bile acid concentrations (Table 1). Albeit variations in the concentration of bile acids, the overall trend is that the highest concentrations in rats are detected in the distal part of the small intestine. Compared to phospholipid values in the human small intestine (Table 1), the concentration in rats is in a similar concentration range. However, the bile acid concentration in the small intestine of rats is 
much higher than in the fasted human small intestine (Table 1). An explanation for this difference can be the continuous flow of bile from the bile duct in rats, due to the absence of a gallbladder in rats [15].

Compared to the osmolalities detected in the present study (Fig. 2C), the osmolality in human GI fluids has been reported to be slightly lower (Table 1). For fluids simulating the human gastric and intestinal conditions in the fasted state, osmolalities of 120 and $180 \mathrm{mOsm} / \mathrm{kg}$ are traditionally applied [9]. These values are notably lower than the values measured in the stomach and proximal small intestine of fasted rats in the current study (230 and $312 \mathrm{mOsm} / \mathrm{kg}$, respectively).

\subsection{MALDI MSI on sections from the small intestine}

In addition to determination of the total concentrations of bile acids and phospholipids (Section 3.2), the location of different bile acids and phospholipid classes were investigated by MALDI MSI of a cross section of the small intestine $(10 \mathrm{~cm}$ distal to the stomach) from a fasted rat.

Cholic acid and its conjugates have shown to be the most dominating bile acid in intestinal fluids in humans and rats [28], and especially sodium taurocholate has been preferred as bile acid for the preparation of biorelevant media $[9,10,28]$. Thus, the presence of cholic acid, taurocholic acid and glycocholic acid, was investigated in a cross section of a rat small intestine $10 \mathrm{~cm}$ distal to the stomach (Fig. 3).
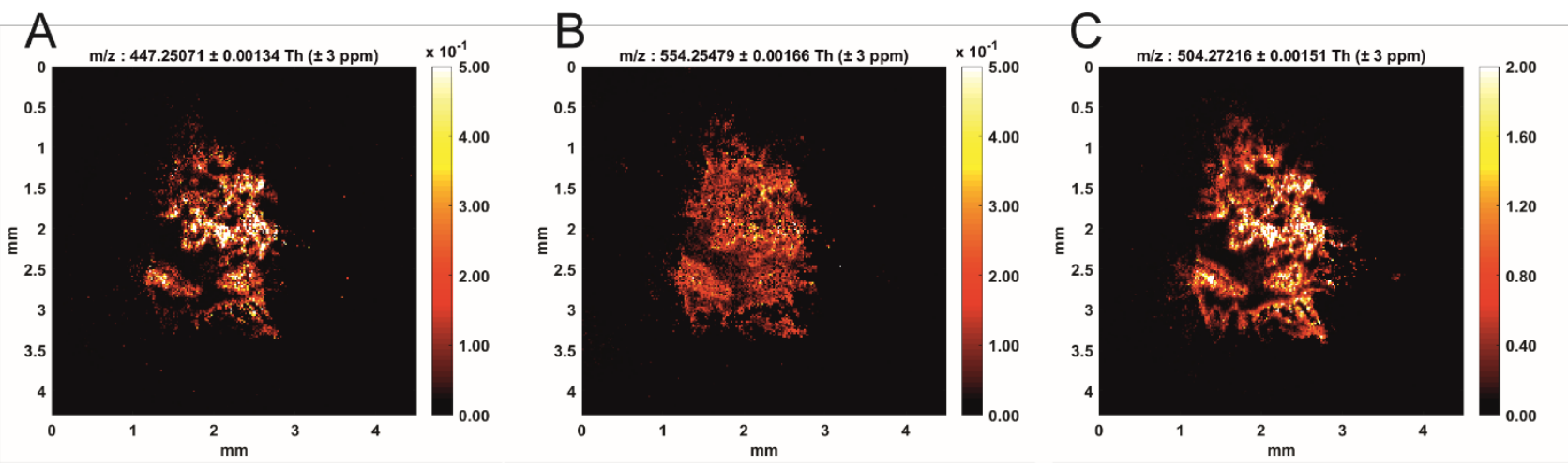
Figure 3: MALDI MSI images of a cross section of a rat small intestine, $10 \mathrm{~cm}$ distal to the stomach. A: Cholic acid (K $\mathrm{K}^{+}$ adduct, $m / z$ 447.2507), B: taurocholic acid ( $\mathrm{K}^{+}$adduct, $m / z$ 554.2548) and C: glycocholic acid (K ${ }^{+}$adduct, $m / z$ 504.2722).

Both cholic acid, taurocholic acid and glycocholic acid were present in the lumen of the GI tract (Fig. 3A, B and C). As previously described, Yang et al. [20] recently determined taurine conjugated bile acids to be the most abundant type in bile from rats, whereas the unconjugated cholic acid play a dominant role in the small intestine.
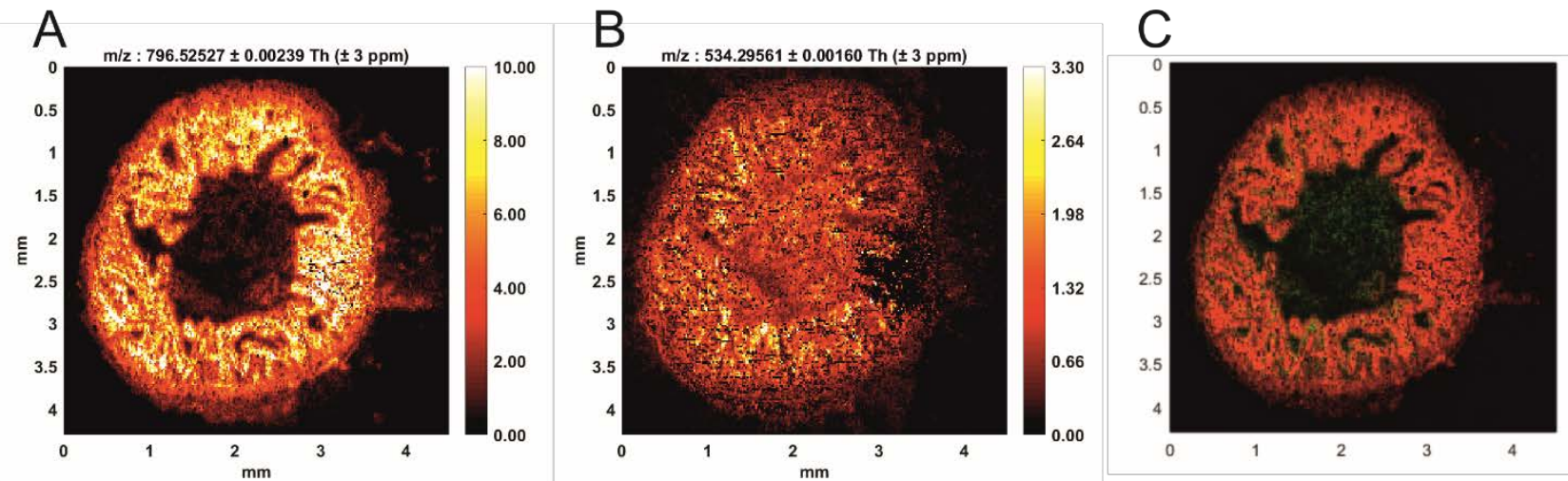

Figure 4: MALDI MSI images of a cross section of a rat small intestine, $10 \mathrm{~cm}$ distal to the stomach. A: Phosphatidylcholine 34:2, B: Lysophosphatidylcholine 16:0 and C: Coloured overlay with phosphatidylcholine $34: 2$ shown in red and lysophosphatidylcholine 16:0 shown in green. The images show the presence of lysophosphatidylcholine 16:0 in the entire cross section and phosphatidylcholine 34:2 primarily present in the intestinal wall. Both compounds were predominantly ionised, and therefore shown, as their potassium adducts.

As seen in Fig. 4A, phosphatidylcholine 34:2 was found to be mainly present in the wall of the GI tract or in the mucus, and to a less extent in the lumen, whereas lysophosphatidylcholine 16:0 was quite homogeneously distributed in the entire cross section of the GI tract (Fig. 4B). In the upper cross sections ( $0-10 \mathrm{~cm}$ distal to the stomach) of the intestine, similar distributions were also observed for several other phosphatidylcholine and lysophosphatidylcholine species than the phosphatidylcholine 34:2 and lysophosphatidylcholine 16:0 shown here. However, lysophosphatidylcholine was not observed in the lumen further down in the small intestine (Appendix A). 
These findings illustrated in Fig. 4 are supported by previous investigations [29, 30], where lysophosphatidylcholine was determined to be the most predominant phospholipid class in fasted intestinal fluids from humans. In bile, the most predominant phospholipid class is phosphatidylcholine, however it is hydrolysed to lysophosphatidylcholine by phospholipases in the small intestine, making lysophosphatidylcholine the most abundant phospholipid class in the small intestine [7].

\subsection{Development of biorelevant media and two-step in vitro model}

RGM and RIM were designed to simulate the conditions observed in the GI tract of fasted rats, regarding $\mathrm{pH}$ and levels of bile acids, phospholipids and osmolality (Sections 3.1 and 3.2). RIM was composed to simulate the conditions in the proximal part of the small intestine. The media were combined in a two-step in vitro dissolution model, to study release and dissolution of drug with a $\mu$ DISS Profiler ${ }^{\mathrm{TM}}$ in both media consecutively. The gastric step was run for 30 min, where after the medium was changed to simulate intestinal conditions, by addition of $5 \mathrm{~mL}$ concentrated bile buffer (Table 3).

Lysophosphatidylcholine was selected to simulate phospholipids in the developed media, since the MALDI MSI results revealed a more pronounced presence of lysophosphatidylcholine in the lumen of the upper part of the small intestine, compared to phosphatidylcholine (Section 3.3). Traditionally phosphate buffers have been widely used to control $\mathrm{pH}$, but they have low buffering capacity above pH 7.5 and also a tendency to precipitate cations [31]. Thus, HEPES (pKa $=7.55$, [31]) was selected as buffering agent in the developed media.

Table 3: Composition of the developed biorelevant media and concentrated bile buffer. The composition of the bile buffer was calculated so that mixing RGM and bile buffer in a ratio of 2 to 1 would result in a biorelevant medium with the composition of RIM. 


\begin{tabular}{lccc}
\hline & RGM & RIM & Bile buffer \\
\hline Properties & $2.4^{*}$ & & \\
\hline $\mathrm{pH}$ & 230 & 7.5 & 7.5 \\
Osmolality (mOsm/kg) & 1.6 & 312 & 476 \\
BS:PL ratio & & 6.5 & 7.3 \\
\hline Composition & 4 & - & - \\
\hline HCl (mM) & - & 100 & 300 \\
HEPES (mM) & 1.3 & 24.1 & 69.7 \\
Sodium taurocholate (mM) & 0.8 & 3.7 & 9.5 \\
Lysophosphatidylcholine (mM) & 111.7 & 119.6 & 139.4 \\
Sodium chloride (mM) & q.s. & q.s. & q.s. \\
NaOH/HCl (pH adjustment) & * & & \\
\hline
\end{tabular}

*mean value of the $\mathrm{pH}$ in the forestomach and the glandular stomach.

\subsection{Evaluation of the biorelevant media and the two-step in vitro model}

The developed media (Table 3) were applied in a $\mu$ DISS Profiler ${ }^{\mathrm{TM}}$ to study the dissolution of crystalline furosemide acid and ASSF. The dissolution of the two furosemide forms has previously been evaluated in a biorelevant medium simulating human intestinal conditions (pH 6.5 and $12.5 \mathrm{mM}$ of total surfactant concentration) [5]. However, despite large differences in the in vitro dissolution of the furosemide forms, Nielsen et al. experienced no significant difference of their performance in vivo (Fig. 6) in rats. As an application of the developed media, it was aimed to improve the in vitro 348 in vivo relation, by performing similar in vitro dissolution experiments in the newly developed 349 biorelevant media specifically developed to simulate rats. 

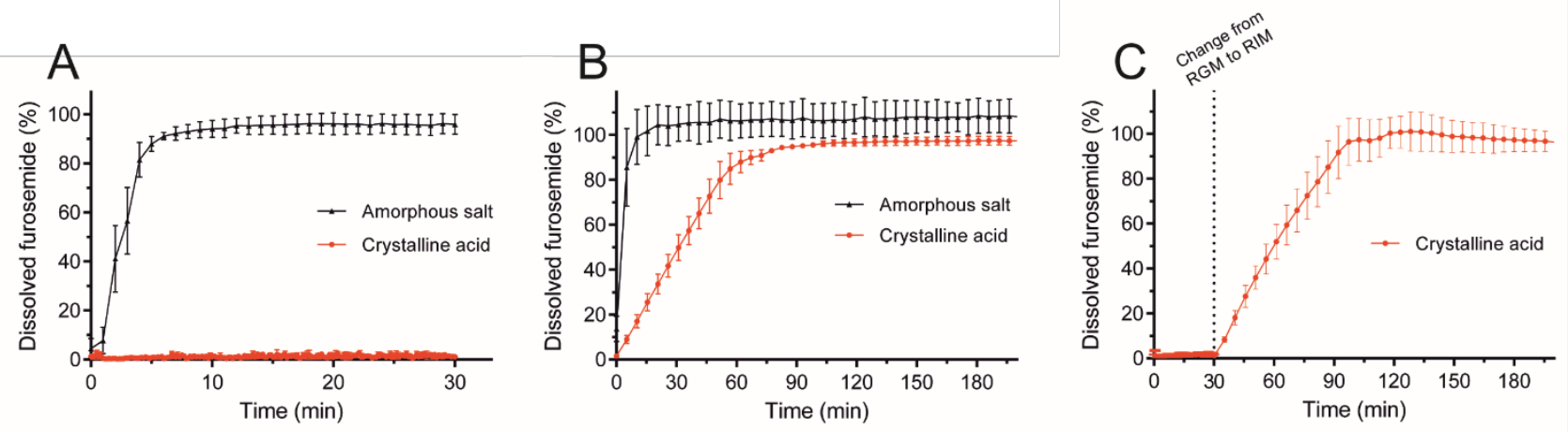

Figure 5: The percentage of furosemide dissolved as a function of time. The dissolution was measured in A: RGM, B: RIM and C: Two-step model including a 30 min gastric step (RGM) before changing the dissolution medium to RIM (mean $\pm \mathrm{SD}, \mathrm{n}=3-5$ ).

In RGM, a dissolution of $87.9 \pm 3.0 \%$ was observed for ASSF within the first 5 min of the experiment, followed by a plateau of $95.6 \pm 3.7 \%$ dissolved after 15 min (Fig. 5A). In contrast, only $1.1 \pm 0.5 \%$ of the loaded amount of crystalline acid was dissolved within 30 min in RGM. This indicates that the crystalline acid is very poorly soluble in RGM, which correlates well with furosemides $\mathrm{pK}_{\mathrm{a}}$-value of 4.2 [6]. Amorphisation and salt formation are both well-known tools to increase the solubility of a compound [32], which appears to be the case for furosemide in RGM as well (Fig. 5A). In RIM, the crystalline acid was almost completely dissolved (94.9 $\pm 1.1 \%$ ) after approximately 90 min in RIM, and this was still slower than for ASSF where $99.3 \pm 13.0 \%$ was dissolved after 10 min (Fig. 5B).

To evaluate the two-step dissolution model, the dissolution of crystalline furosemide acid was further investigated in the in vitro model with the two media incorporated (Fig. 5C). ASSF was not evaluated in the two-step dissolution model due to the fast dissolution of ASSF in RGM (90 \% in 5 min). The dissolution of furosemide crystalline acid in the two-step model showed dissolution trends similar to the dissolution in the individual media. Very limited dissolution was observed in RGM (1.2 $\pm 0.6 \%)$, but $100.5 \pm 6.5 \%$ crystalline furosemide acid was dissolved after $90 \mathrm{~min}$ after changing the medium to RIM (120 min in total).

Comparing the in vitro dissolution results in the developed media (Fig. 5) with the in vitro and in vivo results published by Nielsen et al. [5] (Fig. 6A and B, respectively) reveals interesting trends. By 

approximately $18 \%$ after 90 min to $95 \%$ after 90 min, for the crystalline acid.
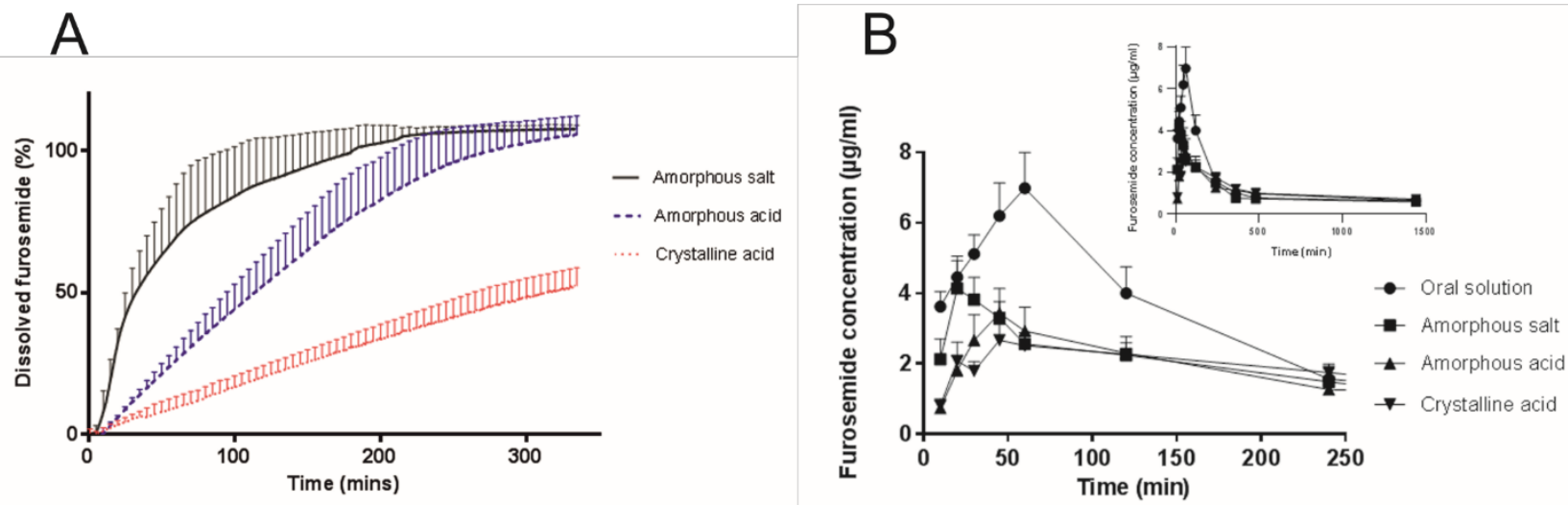

Figure 6: Previously published furosemide in vitro and in vivo data. A: The percentage of dissolved furosemide as a function of time (min) studied by dissolution in human simulated intestinal fluid using a $\mu$ DISS Profiler ${ }^{\mathrm{TM}}$. B: Furosemide concentration in plasma over time studied by oral gavage in rats. Reprinted from [5] with permission.

This difference can be explained by the physicochemical properties of furosemide. Increasing the $\mathrm{pH}$ one unit away from the $\mathrm{pK}_{\mathrm{a}}$-value (from $\mathrm{pH} 6.5$ to 7.5 ) will increase the concentration of the ionised form of furosemide by a factor 10, resulting in a higher solubility in water. Additionally, increasing the concentration of bile acids and phospholipids have shown to increase the solubility of many poorly water-soluble compounds [33, 34]. Hence, the increase in total concentration of bile acids and phospholipids from $12.5 \mathrm{mM}$ in the human intestinal medium to $27.8 \mathrm{mM}$ in RIM could also contribute to the increased dissolution.

Since both crystalline furosemide acid and ASSF reach more than $90 \%$ dissolved after the first 90 min in RIM (Fig. 5B), it seems more reasonable to predict that there is no significant difference between the in vivo performances of the two compounds. This was also the result from the in vivo study in rats performed by Nielsen et al. (Fig. 6B). Based on this comparison, it is, in the present case, more predictive to apply RIM than human simulated intestinal fluid for in vitro dissolution studies prior to in vivo studies in rats. 


\section{Conclusion}

The $\mathrm{pH}$ and osmolality were found to increase throughout the GI tract of fasted rats and was significant different from human values. Bile acids and phospholipids were present in high concentrations in the proximal small intestine, and the bile acid concentration doubled in the distal part. From MALDI MSI of an intestinal cross section from a fasted rat, the bile acids cholic acid, glycocholic acid and taurocholic acid were found to be present in the lumen of the small intestine. Phosphatidylcholine species were mainly detected in the intestinal wall or mucus of the small intestine, whereas lysophosphatidylcholine species were detected in the whole cross section including the lumen of the upper part of the small intestine. Based on these observations, a two-step in vitro dissolution model, with biorelevant media simulating GI fluids of rats, was developed. The incorporation of the media simulating GI fluids in fasted rats provided better prediction of the in vivo performance of furosemide in rats compared to human biorelevant media. This indicates that the model is a promising tool to in vitro evaluation of a compound before proceeding to in vivo studies in rats. However, similar evaluations of other model drugs would be needed to evaluate the full potential of the model.

\section{Acknowledgements}

The Danish National Research Foundation (Project DNRF122) and Villum Foundation's Center (Grant No. 9301) for Intelligent Drug Delivery and Sensing Using Microcontainers and Nanomechanics (IDUN) is acknowledged. The authors would also like to acknowledge Nanna Bild, Department of Health Technology, Technical University of Denmark, for help with the drawings.

Declaration of interests: None. 


\section{References}

1. C. J. Andreas, J. Rosenberger, J. Butler, P. Augustijns, M. McAllister, B. Abrahamsson, J. Dressman, Introduction to the OrBiTo decision tree to select the most appropriate in vitro methodology for release testing of solid oral dosage forms during development, Eur. J. Pharm. Biopharm. 130 (2018) p. 207-213.

2. J. Butler, B. Hens, M. Vertzoni, J. Brouwers, P. Berben, J. Dressman, C. J. Andreas, K. J. Schaefer, J. Mann, M. McAllister, M. Jamei, E. Kostewicz, F. Kesisoglou, P. Langguth, M. Minekus, A. Mullertz, R. Schilderink, M. Koziolek, P. Jedamzik, W. Weitschies, C. Reppas, P. Augustijns, In vitro models for the prediction of in vivo performance of oral dosage forms: Recent progress from partnership through the IMI OrBiTo collaboration, Eur. J. Pharm. Biopharm. 136 (2019) p. 70-83.

3. H. Higashino, T. Hasegawa, M. Yamamoto, R. Matsui, Y. Masaoka, M. Kataoka, S. Sakuma, S. Yamashita, In vitro-in vivo correlation of the effect of supersaturation on the intestinal absorption of BCS Class 2 drugs, Mol. Pharmaceutics 11 (2014) p. 746-754.

4. T. Tran, S. D. V. S. Siqueira, H. Amenitsch, A. Müllertz, T. Rades, In vitro and in vivo performance of monoacyl phospholipid-based self-emulsifying drug delivery systems, J. Controlled Release 255 (2017) p. 45-53.

5. L. H. Nielsen, S. Gordon, R. Holm, A. Selen, T. Rades, A. Mullertz, Preparation of an amorphous sodium furosemide salt improves solubility and dissolution rate and leads to a faster Tmax after oral dosing to rats, Eur. J. Pharm. Biopharm. 85 (2013) p. 942-951.

6. P. Augustijns, B. Wuyts, B. Hens, P. Annaert, J. Butler, J. Brouwers, A review of drug solubility in human intestinal fluids: implications for the prediction of oral absorption, Eur. J. Pharm. Sci. 57 (2014) p. 322-332.

7. A. Fuchs, J. B. Dressman, Composition and Physicochemical Properties of Fasted State Human Duodenal and Jejunal Fluid: A Critical Evaluation of the Available Data, J. Pharm. Sci. 103 (2014) p. 3398-3411.

8. P. B. Pedersen, P. Vilmann, D. Bar-Shalom, A. Mullertz, S. Baldursdottir, Characterization of fasted human gastric fluid for relevant rheological parameters and gastric lipase activities, Eur. J. Pharm. Biopharm. 85 (2013) p. 958-965.

9. E. Jantratid, N. Janssen, C. Reppas, J. B. Dressman, Dissolution media simulating conditions in the proximal human gastrointestinal tract: An update, Pharm. Res. 25 (2008) p. 1663-1676.

10. A. Fuchs, M. Leigh, B. Kloefer, J. B. Dressman, Advances in the design of fasted state simulating intestinal fluids: FaSSIF-V3, Eur. J. Pharm. Biopharm. 94 (2015) p. 229-240.

11. C. Markopoulos, C. J. Andreas, M. Vertzoni, J. Dressman, C. Reppas, In-vitro simulation of luminal conditions for evaluation of performance of oral drug products: Choosing the appropriate test media, Eur. J. Pharm. Biopharm. 93 (2015) p. 173-182.

12. Y. Tanaka, T. Baba, K. Tagawa, R. Waki, S. Nagata, Prediction of oral absorption of lowsolubility drugs by using rat simulated gastrointestinal fluids: the importance of regional differences in membrane permeability and solubility, J. Pharm. Pharm. Sci. 17 (2014) p. 106120.

13. Z. Warnken, M. Puppolo, J. Hughey, I. Duarte, S. Jansen-Varnum, In Vitro-In Vivo Correlations of Carbamazepine Nanodispersions for Application in Formulation Development, J. Pharm. Sci. 107 (2018) p. 453-465.

14. C. A. Bergstrom, R. Holm, S. A. Jorgensen, S. B. Andersson, P. Artursson, S. Beato, A. Borde, K. Box, M. Brewster, J. Dressman, K. I. Feng, G. Halbert, E. Kostewicz, M. McAllister, U. Muenster, J. Thinnes, R. Taylor, A. Mullertz, Early pharmaceutical profiling to predict oral drug absorption: current status and unmet needs, Eur. J. Pharm. Sci. 57 (2014) p. 173-199. 
15. Y. Tanaka, T. Hara, R. Waki, S. Nagata, Regional differences in the components of luminal water from rat gastrointestinal tract and comparison with other species, J. Pharm. Pharm. Sci. 15 (2012) p. 510-518.

16. E. L. McConnell, A. W. Basit, S. Murdan, Measurements of rat and mouse gastrointestinal $\mathrm{pH}$, fluid and lymphoid tissue, and implications for in-vivo experiments, J. Pharm. Pharmacol. 60 (2008) p. 63-70.

17. F. W. Ward, M. E. Coates, Gastrointestinal $\mathrm{pH}$ measurement in rats: influence of the microbial flora, diet and fasting, Lab. Anim. 21 (1987) p. 216-222.

18. R. Holm, A. Mullertz, H. Mu, Bile salts and their importance for drug absorption, Int. J. Pharm. 453 (2013) p. 44-55.

19. D. Riethorst, R. Mols, G. Duchateau, J. Tack, J. Brouwers, P. Augustijns, Characterization of Human Duodenal Fluids in Fasted and Fed State Conditions, J. Pharm. Sci. 105 (2016) p. 673681.

20. T. Yang, T. Shu, G. Liu, H. Mei, X. Zhu, X. Huang, L. Zhang, Z. Jiang, Quantitative profiling of 19 bile acids in rat plasma, liver, bile and different intestinal section contents to investigate bile acid homeostasis and the application of temporal variation of endogenous bile acids, $\mathrm{J}$. Steroid Biochem. Mol. Biol. 172 (2017) p. 69-78.

21. A. Rompp, B. Spengler, Mass spectrometry imaging with high resolution in mass and space, Histochem. Cell Biol. 139 (2013) p. 759-783.

22. E. Marxen, L. Jin, J. Jacobsen, C. Janfelt, B. Hyrup, J. A. Nicolazzo, Effect of Permeation Enhancers on the Buccal Permeability of Nicotine: Ex vivo Transport Studies Complemented by MALDI MS Imaging, Pharm. Res. 35 (2018) p. 70.

23. S. E. Hansen, E. Marxen, C. Janfelt, J. Jacobsen, Buccal delivery of small molecules - Impact of levulinic acid, oleic acid, sodium dodecyl sulfate and hypotonicity on ex vivo permeability and spatial distribution in mucosa, Eur. J. Pharm. Biopharm. 133 (2018) p. 250-257.

24. E. Wenande, U. H. Olesen, M. M. Nielsen, C. Janfelt, S. H. Hansen, R. R. Anderson, M. Haedersdal, Fractional laser-assisted topical delivery leads to enhanced, accelerated and deeper cutaneous 5-fluorouracil uptake, Expert Opin. Drug Delivery 14 (2017) p. 307-317.

25. T. Schramm, A. Hester, I. Klinkert, J. P. Both, R. M. Heeren, A. Brunelle, O. Laprevote, N. Desbenoit, M. F. Robbe, M. Stoeckli, B. Spengler, A. Rompp, imzML--a common data format for the flexible exchange and processing of mass spectrometry imaging data, J. Proteomics 75 (2012) p. 5106-5110.

26. G. Robichaud, K. P. Garrard, J. A. Barry, D. C. Muddiman, MSiReader: an open-source interface to view and analyze high resolving power MS imaging files on Matlab platform, J. Am. Soc. Mass Spectrom. 24 (2013) p. 718-721.

27. T. T. Kararli, Comparison of the gastrointestinal anatomy, physiology, and biochemistry of humans and commonly used laboratory animals, Biopharm. Drug Dispos. 16 (1995) p. 351380.

28. K. Kleberg, J. Jacobsen, A. Mullertz, Characterising the behaviour of poorly water soluble drugs in the intestine: application of biorelevant media for solubility, dissolution and transport studies, J. Pharm. Pharmacol. 62 (2010) p. 1656-1668.

29. D. Psachoulias, M. Vertzoni, K. Goumas, V. Kalioras, S. Beato, J. Butler, C. Reppas, Precipitation in and supersaturation of contents of the upper small intestine after administration of two weak bases to fasted adults, Pharm. Res. 28 (2011) p. 3145-3158.

30. E. Persson, L. Lofgren, G. Hansson, B. Abrahamsson, H. Lennernas, R. Nilsson, Simultaneous assessment of lipid classes and bile acids in human intestinal fluid by solidphase extraction and HPLC methods, J. Lipid Res. 48 (2007) p. 242-251. 
31. N. E. Good, G. D. Winget, W. Winter, T. N. Connolly, S. Izawa, R. M. M. Singh, Hydrogen Ion Buffers for Biological Research*, Biochemistry 5 (1966) p. 467-477.

32. Y. Perrie, T. Rades, Pharmaceutics - Drug delivery and targeting, Pharmaceutical Press, London, 2012, p. 28 and 47.

33. J. H. Fagerberg, O. Tsinman, N. Sun, K. Tsinman, A. Avdeef, C. A. Bergstrom, Dissolution rate and apparent solubility of poorly soluble drugs in biorelevant dissolution media, Mol. Pharmaceutics 7 (2010) p. 1419-1430.

34. C. M. Madsen, K. I. Feng, A. Leithead, N. Canfield, S. A. Jorgensen, A. Mullertz, T. Rades, Effect of composition of simulated intestinal media on the solubility of poorly soluble compounds investigated by design of experiments, Eur. J. Pharm. Sci. 111 (2018) p. 311-319. 


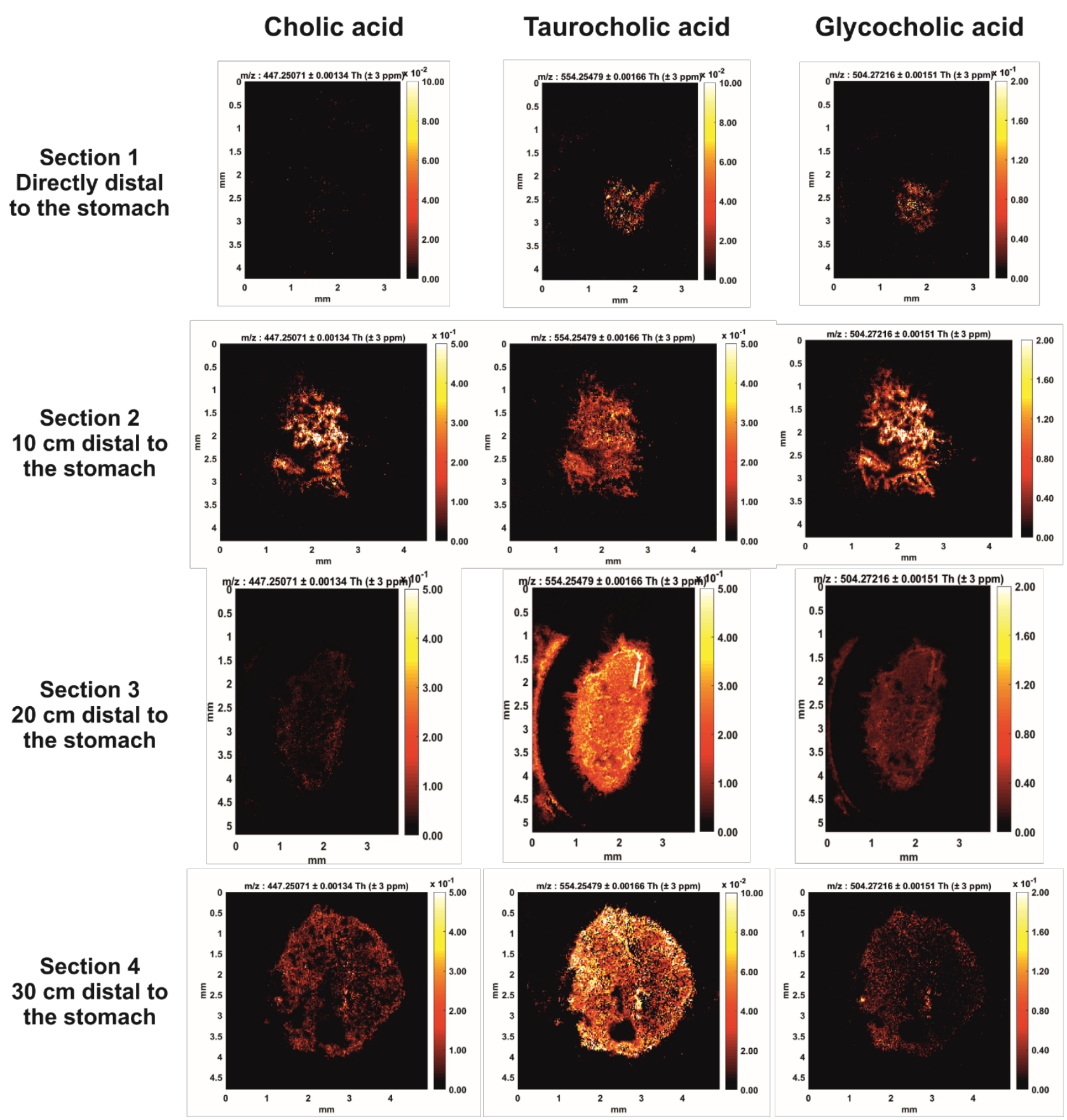

Figure A.1: MALDI MSI images showing the location of three bile acids in four cross sections of a rat small intestine; section $1-4\left(0,10,20\right.$ and $30 \mathrm{~cm}$ distal to the stomach, respectively). ). Left coloumn shows cholic acid ( $\mathrm{K}^{+}$adduct, $\mathrm{m} / \mathrm{z}$ 447.2507), middle column shows glycocholic acid ( $\mathrm{K}^{+}$adduct, $\mathrm{m} / \mathrm{z}$ 504.2722) and right column shows taurocholic acid ( $\mathrm{K}^{+}$adduct, $m / z$ 554.2548). 


\section{Phosphatidylcholine Lysophosphatidylcholine 34:2 16:0}

Section 1 Directly distal to the stomach
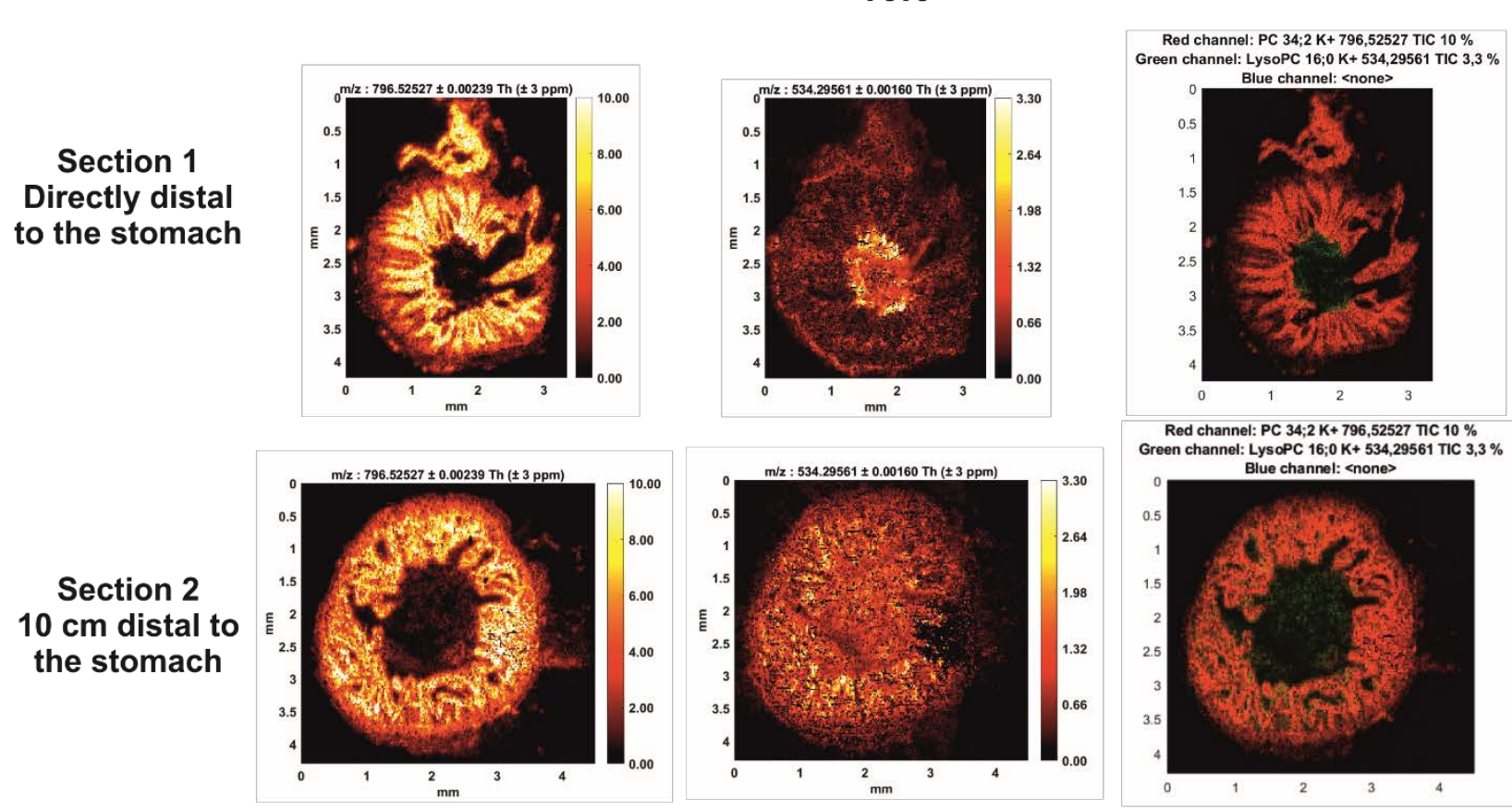

Green channel: LysoPC 16;0 K+ 534,29561 TIC 3,3\% Green channel: LysoPC $16 ; 0 \mathrm{~K}+534,29$
Blue channel: $\langle$ none>
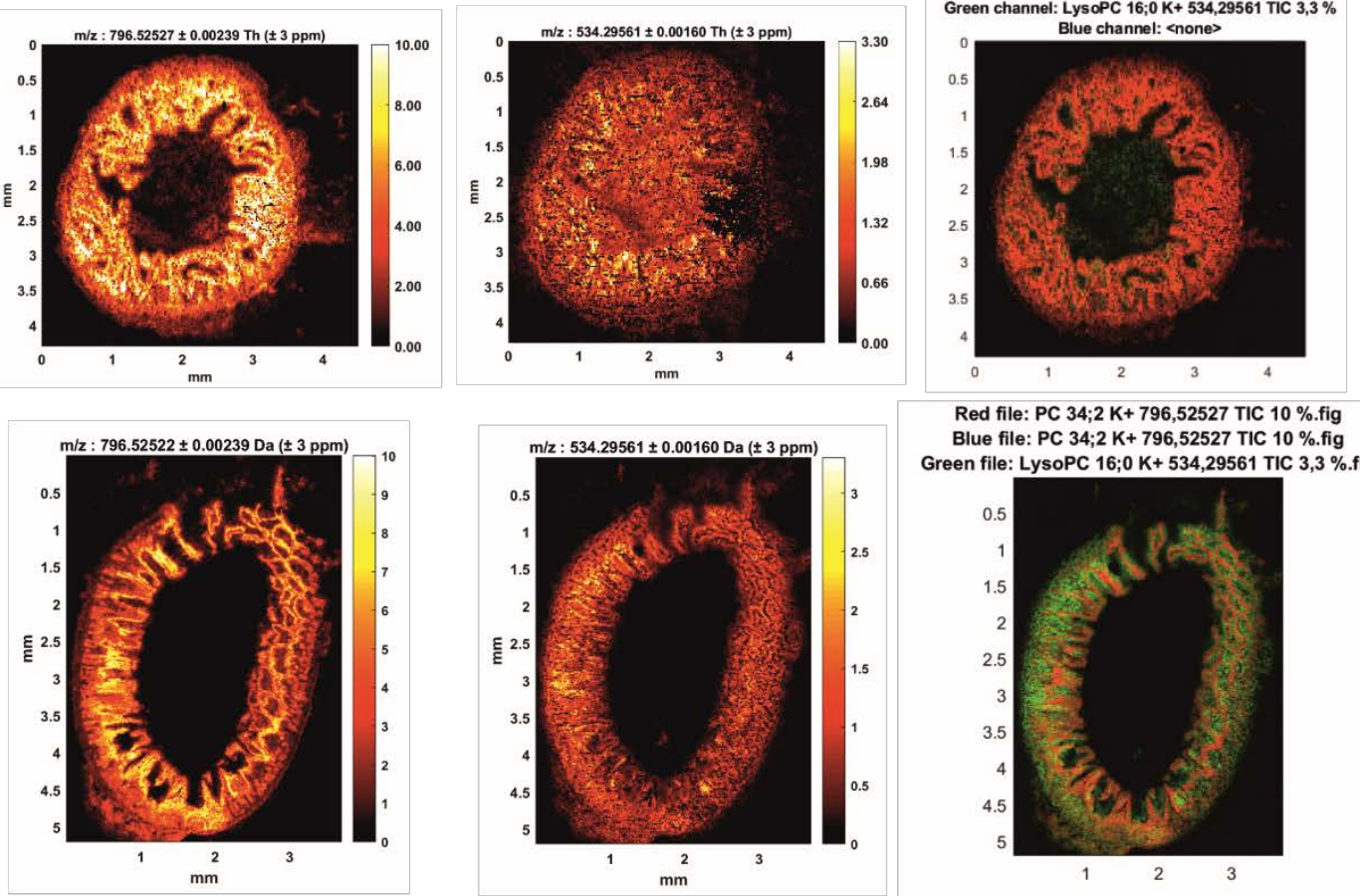

Red file: PC 34;2 K+ 796,52527 TIC $10 \%$.fig Blue file: PC 34;2 K+ 796,52527 TIC $10 \%$.fig Green file: LysoPC 16;0 K+534,29561 TIC 3,3\%.fig

\section{Section 3}

$20 \mathrm{~cm}$ distal to the stomach

\section{Section 4 $30 \mathrm{~cm}$ distal to the stomach}
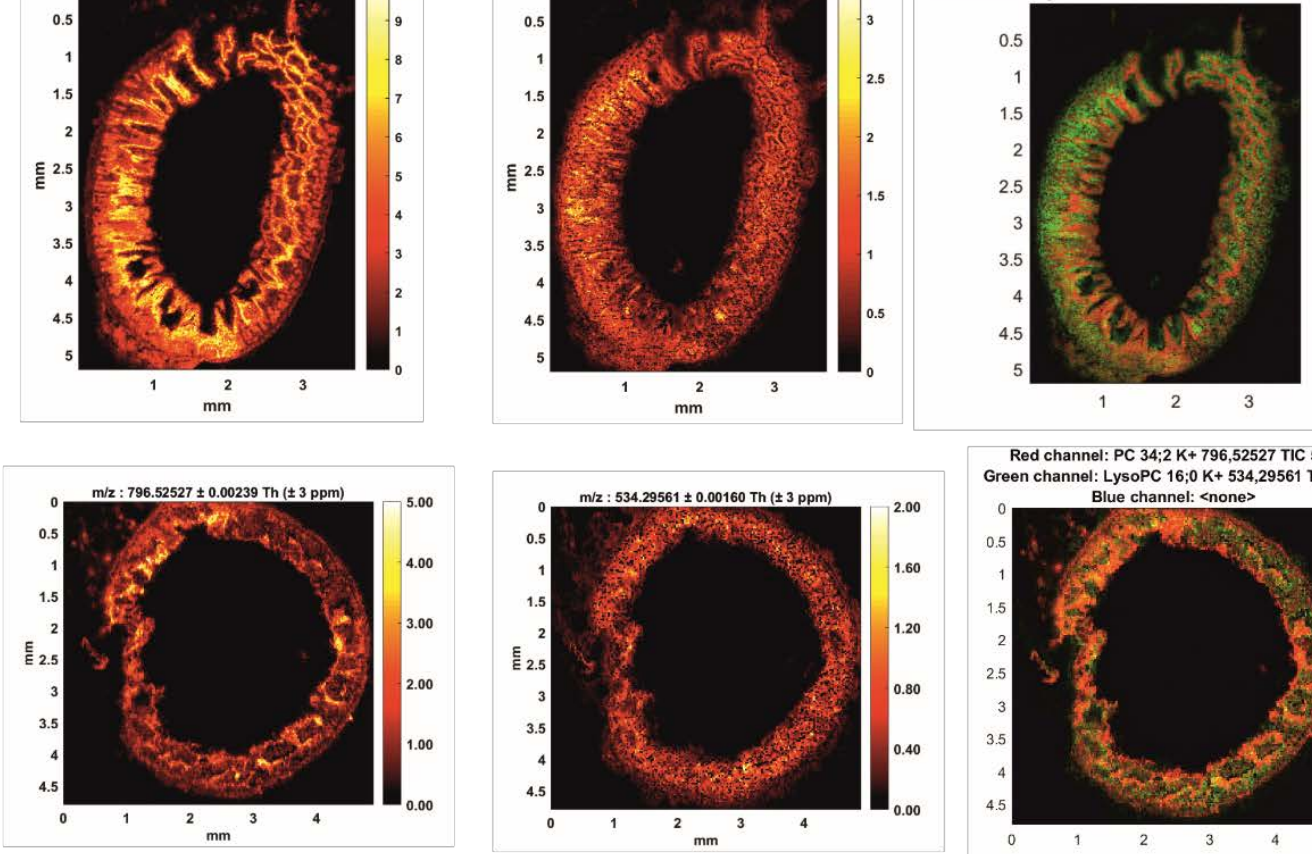
Red channel: PC 34; 2 K+ 796,52527 TIC $5 \%$
Green channel: LysoPC $16 ; 0 \mathrm{~K}+534,29561$ TIC $2 \%$

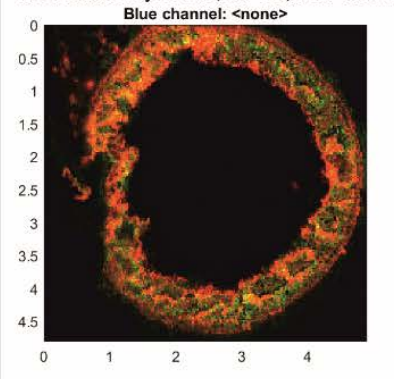

Figure A.2: MALDI MSI images of four cross sections of a rat small intestine; section $1-4(0,10,20$ and $30 \mathrm{~cm}$ distal to the stomach, respectively). Left column shows phosphatidylcholine 34:2 ( $\mathrm{K}^{+}$adduct, $\mathrm{m} / \mathrm{z}$ 796.5253), middle column shows lysophosphatidylcholine 16:0 ( $\mathrm{K}^{+}$adduct, $\mathrm{m} / \mathrm{z}$ 534.2956) and the right column shows an overlay with phosphatidylcholine 34:2 shown in red and lysophosphatidylcholine 16:0 shown in green. 